\title{
Newtonian Dynamics from the principle of Maximum Caliber
}

\author{
Diego González:* Sergio Davis \\ Grupo de Nanomateriales, Departamento de Física, \\ Facultad de Ciencias, Universidad de Chile, Casilla 653, Santiago, Chile
}

(Dated: August 28, 2018)

\begin{abstract}
The foundations of Statistical Mechanics can be recovered almost in their entirety from the Principle of Maximum Entropy. In this work we show that its non-equilibrium generalization, the Principle of Maximum Caliber (Jaynes, 1980), when applied to the unknown trajectory followed by a particle, leads to Newton's second law under two quite intuitive assumptions (the expected square displacement in one step and the spatial probability distribution of the particle are known at all times). Our derivation explicitly highlights the role of mass as an emergent measure of the fluctuations in velocity (inertia) and the origin of potential energy as a manifestation of spatial correlations. According to our findings, the application of Newton's equations is not limited to mechanical systems, and therefore could be used in modelling ecological, financial and biological systems, among others.

PACS numbers:
\end{abstract}

${ }^{*}$ Electronic address: dgonzalez@gnm.cl

$\dagger$ Electronic address: sdavis@gnm.cl

${ }^{\ddagger}$ Electronic address: gonzalo@macul.ciencias.uchile.cl 


\section{INTRODUCTION}

In 1957, E. T. Jaynes [1] postulated that Statistical Mechanics has to be understood, not as a physical theory in the same footing as, say, classical mechanics or electromagnetism, but as an application of statistical inference on a system with macroscopically large numbers of degrees of freedom. The question was reversed from "given the microscopic evolution of the system, what is the probability distribution for the macroscopic quantities?" to "given a few known macroscopic properties, what are the possible microstates compatible with said knowledge?". The answer, as initially proposed by Gibbs, was the probability distribution with maximum entropy $S=-\sum_{i} P_{i} \ln P_{i}$ subjected to constraints reflecting the known macroscopic properties. Jaynes, after the work of Shannon in information theory, realized that this procedure (maximization of $S$ constrained only by the known information) is not limited to Statistical Mechanics but a valid principle in any problem of statistical inference. Due to the uniqueness of Shannon's entropy in characterizing uncertainty it is the most unbiased procedure for the construction of statistical models. Later, it has been axiomatically derived [2, 3] from requirements of internal consistency.

The principle of Maximum Caliber [4] generalizes the idea of Maximum Entropy to dynamical systems, including time explicitly. For this, we now ask for the possible microscopical trajectories compatible with known information. The result is the probability distribution of trajectories $P[x(t) \mid H]$ which maximizes the Shannon entropy, now defined as the functional integral

$$
\mathcal{S}=-\int D x(t) P[x(t) \mid H] \ln P[x(t) \mid H] .
$$

Maximum Caliber has been applied recently to discrete dynamics [5] and earlier to derive the Fokker-Planck equations [6] and the Markov process formalism [7].

Every Maximum Caliber solution predicts that the most probable trajectory is the one that extremizes some functional (analogous to an "action" in classical mechanics), in the same way that every Maximum Entropy solution predicts that the most probable microstate is the one that extremizes some function which is a combination of all the constraints imposed. This leads to the question: without introducing the Lagrangian of classical mechanics explicitly, could it "emerge" naturally from simpler constraints in a Maximum Caliber problem? 
Newtonian dynamics has been previously derived from information-geometric arguments [8] leading to the idea of entropic dynamics. This idea is based on the assumption of an irreducible uncertainty in the position of a particle, implying an information metric for space from which Newton's second law naturally emerges. Caticha's derivation is founded on the Maximum Entropy principle, suitably modified to update a prior distribution under new constraints.

In this work we show that if we use the Maximum Caliber principle to find the unknown trajectory of a particle, there are two general conditions that lead to Newton's second law, namely that (a) the expected square displacement per step is known at all times, and (b) that the time-independent probability of finding the particle at any coordinate is also known. Knowledge of both (a) and (b) leads to Newton's second law in expectation over trajectories, and what is perhaps more interesting, any dynamical system not following Newton's second law has to violate at least one of these assumptions.

\section{THE MAXIMUM ENTROPY AND MAXIMUM CALIBER FORMALISM}

Consider a system with $N$ degrees of freedom, whose states are denoted by vectors $\vec{x}=$ $\left(x_{1}, \ldots, x_{N}\right)$. Suppose the expectation values of $M$ functions $f_{i}(\vec{x})$ are known. Maximization of the Shannon entropy leads to the MaxEnt model

$$
P(\vec{x} \mid H)=\frac{1}{Z(\vec{\lambda})} \exp \left(-\sum_{k=1}^{M} \lambda_{k} f_{k}(\vec{x})\right),
$$

where the value of the Lagrange multipliers $\lambda_{k}$ needed to impose the $M$ constraints can be determined from

$$
-\frac{\partial}{\partial \lambda_{k}} \ln Z(\vec{\lambda})=\left\langle f_{k}(\vec{x})\right\rangle .
$$

This nonlinear equation is usually impractical to solve, as it needs the partition function explicitly. It has been recently shown that [9] for the Lagrange multipliers the equality

$$
\langle\nabla \cdot \vec{v}\rangle=\sum_{k=1}^{M} \lambda_{k}\left\langle\vec{v} \cdot \nabla f_{k}\right\rangle
$$

holds, with $\vec{v}$ an arbitrary differentiable vector field, and this provides a linear system of equations for $\vec{\lambda}$. 
Now, suppose $N$ is infinitely large, the state vector $\vec{x}$ becomes a function of a continuous parameter, let us call it $t$, i.e., a parameterized trajectory. The probability distribution functional for the different possible trajectories is (from Eq. 2 in the $N \rightarrow \infty$ limit),

$$
P[x(t) \mid H]=\frac{1}{Z[\lambda(t)]} e^{-\int d t \lambda(t) f[x(t) ; t]},
$$

where, similarly to Eq. 3, the Lagrange multiplier function can be obtained from

$$
-\frac{\delta}{\delta \lambda(t)} \ln Z[\lambda(t)]=\langle f[x(t) ; t]\rangle .
$$

If we discretize time, the trajectory $x(t)$ becomes a vector $\vec{x}=\left(x_{0}, \ldots, x_{n-1}\right)$, and the Lagrange function $\lambda(t)$ becomes a vector $\vec{\lambda}=\left(\lambda_{0}, \ldots, \lambda_{n-1}\right)$. In fact, we recover Eqs. 2 and 3. This means we can use Eq. 4 in a discretized Maximum Caliber problem.

\section{DERIVATION OF NEWTON'S SECOND LAW}

Consider a single particle following an unknown trajectory $x(t)$ in one spatial dimension. This can be easily generalized to many particles in arbitrary dimensions, at the cost of overcomplicated notation. We can discretize this trajectory in $n$ steps, such that $x(t)$ now becomes a vector $\vec{x}=\left(x_{0}, \ldots, x_{n-1}\right)$, and then impose the following constraints (expectations are to be interpreted over all possible trajectories)

$$
\begin{array}{r}
\left\langle\left(x_{i}-x_{i-1}\right)^{2}\right\rangle=(\Delta t)^{2} d_{i}{ }^{2} \\
\left\langle\delta\left(x_{i}-X\right)\right\rangle=P\left(x_{i}=X \mid H\right),
\end{array}
$$

for all values of $i$ and $X$. The first constraint recognizes the fact that the expected square displacement in one (possible infinitesimal) step is known for all times, and is equal to an arbitrary function $d_{i}{ }^{2}$ times the time step. We expressed it in this form so that $d_{i}$ can remain finite when taking the limit $\Delta t \rightarrow 0$. The second constraint imposes that the static, time-independent probability distribution for the coordinate $x$ is also known.

The probability distribution function for $\vec{x}$ is

$$
P(\vec{x} \mid H)=\frac{1}{Z(\vec{\lambda})} \exp \left(-\sum_{i=0}^{n-1} \frac{\lambda_{i}}{(\Delta t)^{2}}\left(x_{i}-x_{i-1}\right)^{2}+\sum_{i=0}^{n-1} \int d X \mu(X) \delta\left(x_{i}-X\right)\right)
$$


which, after integrating the Dirac delta function, becomes

$$
P(\vec{x} \mid H)=\frac{1}{Z(\vec{\lambda})} \exp \left(-\sum_{i=0}^{n-1} \frac{\lambda_{i}}{(\Delta t)^{2}}\left(x_{i}-x_{i-1}\right)^{2}+\sum_{i=0}^{n-1} \mu\left(x_{i}\right)\right) .
$$

This is the probability of the particle taking a well-defined discretized trajectory $\vec{x}$, and is precisely the solution of a Maximum Entropy problem with $n$ degrees of freedom and $n$ Lagrange multipliers $\lambda_{i}$ (plus the function $\mu$ ), therefore Eq. 4 holds as

$$
\langle\nabla \cdot \vec{v}(\vec{x})\rangle=\sum_{i=0}^{n-1} \frac{\lambda_{i}}{(\Delta t)^{2}}\left\langle\vec{v}(\vec{x}) \cdot \nabla\left(x_{i}-x_{i-1}\right)^{2}\right\rangle+\sum_{i=0}^{n-1}\left\langle\vec{v}(\vec{x}) \cdot \nabla \mu\left(x_{i}\right)\right\rangle,
$$

with $\vec{v}$ an arbitrary vector field, of our choosing. If we choose $\vec{v}$ such that it has a single component $k$, i.e. $v_{i}=\delta_{i, k} \omega(\vec{x})$ with $\omega$ an arbitrary scalar field, we obtain

$$
\begin{aligned}
\left\langle\frac{\partial \omega}{\partial x_{k}}\right\rangle= & \sum_{i=0}^{n-1} \frac{\lambda_{i}}{(\Delta t)^{2}}\left\langle\omega(\vec{x}) \cdot 2\left(x_{i}-x_{i-1}\right)\left(\delta_{i, k}-\delta_{i-1, k}\right)\right\rangle+\sum_{i=0}^{n-1}\left\langle\omega(\vec{x}) \cdot \mu^{\prime}\left(x_{i}\right) \delta_{i, k}\right\rangle \\
& =\frac{1}{(\Delta t)^{2}}\left\langle 2 \omega(\vec{x})\left[\lambda_{k}\left(x_{k}-x_{k-1}\right)-\lambda_{k+1}\left(x_{k+1}-x_{k}\right)\right]\right\rangle+\left\langle\omega(\vec{x}) \mu^{\prime}\left(x_{k}\right)\right\rangle .
\end{aligned}
$$

But recalling that the discrete forward derivative is

$$
\dot{a}_{i} \approx \frac{a_{i+1}-a_{i}}{\Delta t}
$$

we can write Eq. 13 as

$$
\left\langle\frac{\partial \omega}{\partial x_{k}}\right\rangle=-\left\langle\omega\left(\dot{p}_{k}+\mu^{\prime}\left(x_{k}\right)\right)\right\rangle
$$

where

$$
p_{k}=2 \lambda_{k} \dot{x}_{k}=m_{k} \dot{x}_{k}
$$

Considering $\omega=1$ and defining $\Phi(x)=-\mu(x)$ we finally obtain

$$
\left\langle\dot{p}_{k}\right\rangle=-\left\langle\Phi^{\prime}\left(x_{k}\right)\right\rangle \text {. }
$$

which is a discrete version of Newton's second law with momentum $p(t)=m(t) \dot{x}(t)$ and potential energy $\Phi(x)$.

From this we note that a time-dependent mass $m(t)$ and a potential energy have emerged from the Lagrange multipliers associated with the constraints on the expected square of the 
step and the probability distribution of the coordinate, respectively. Thus we can say the following: whenever the information about the expected square of the step is important, the particle acquires mass, and whenever the information about which regions are more probable in space becomes important, the particle is subjected to a potential energy.

The most probable trajectory for the particle follows a minimum action principle. Indeed, if we replace our definitions of $m_{k}$ and $\Phi\left(x_{k}\right)$ in Eq. 10, we recover in the exponential the classical action

$$
P(\vec{x} \mid H)=\frac{1}{Z} \exp \left(-\sum_{i=0}^{n-1}\left[\frac{1}{2} m_{i} \dot{x}_{i}^{2}-\Phi\left(x_{i}\right)\right]\right)
$$

which in the continuum limit becomes

$$
P[x(t) \mid H]=\frac{1}{Z} \exp \left(-\int d t \mathcal{L}(t)\right)
$$

This tells us that the most probable trajectory is the one that extremizes the classical action with Lagrangian

$$
\mathcal{L}(t)=\frac{p(t)^{2}}{2 m(t)}-\Phi(x(t))
$$

and associated Hamiltonian

$$
\mathcal{H}=\frac{p(t)^{2}}{2 m(t)}+\Phi(x(t)) .
$$

Therefore the most probable trajectory is governed by the canonical formalism of Classical Mechanics. In appendix A we explore the validity of some aspects of the canonical formalism, namely the Poisson bracket, for the expectation over trajectories.

\section{CONCLUDING REMARKS}

We have found that two simple constraints are sufficient to recover Newton's second law in expectation for the probable trajectories of a particle. The first constraint, on the step size as a function of time, leads to the existence of an inertial mass $m(t)$ proportional to the Lagrange multiplier $\lambda(t)$. To understand the meaning of this, remember that for any variational problem solved using Lagrange multipliers, the larger the value of the multiplier, the more restrictive (and therefore more relevant) the constraint. An irrelevant constraint has 
always a vanishing multiplier. As Jaynes [10] (p. 945) clearly states, "The Lagrange multipliers $\lambda_{k}$ in the MAXENT formalism have therefore a deep meaning: $\lambda_{k}$ is the 'potential' of the datum $R_{k}^{\prime}$, that measures how important a constraint it represents."

Now we motivate the following principle: constraints related to conserved quantities are always more relevant. For instance, this explains the fact that the canonical ensemble in equilibrium statistical mechanics is correctly derived just from a single constraint, the energy or expectation of the Hamiltonian, which is an integral of motion. Another illustration is the following: suppose we are trying to recover the trajectory of a particle from information about the distance to a particular point. If this distance is a constant, this is enough to isolate a unique trajectory, the circle. If we only know that the distance varies between $r_{1}$ and $r_{2}$, the number of compatible trajectories will increase with $\Delta r=r_{2}-r_{1}$, thus the strength of the constraint will correspondingly decrease with increasing $\Delta r$.

Given the earlier discussion, the closer $d_{i}^{2}$ is to be a conserved quantity, the more relevant the first constraint is. In this case, $\lambda(t)$ is large and therefore, $m(t)$ is also large. Conversely, if the value of $m$ is small, this means $\lambda(t)$ is small and therefore $d_{i}^{2}$ has larger fluctuations. In the continuous limit it is the instantaneous speed that fluctuates (there is a non-zero acceleration). This embodies the idea of inertia, and is reminiscent of the ideas of Smolin [11] and of Nelson [12] about inertia being inversely proportional to the size of quantum fluctuations.

\section{ACKNOWLEDGEMENTS}

DG gratefully acknowledges the access to resources provided by Grupo de Nano Materiales (Departamento de Física, Facultad de Ciencias, Universidad de Chile).

\section{Appendix A: Canonical coordinates and Poisson brackets}

An interesting question is how much of the formalism of classical mechanics we can recover from Eq. 16. The fact that most of the structure of classical mechanics is contained in the definition and properties of the Poisson bracket, motivates us to search for an operation analogous to this bracket under the Maximum Caliber formalism.

For arbitrary functions $f(x, p)$ and $g(x, p)$ the Poisson bracket is defined as 


$$
\{f, g\}=\frac{\partial f}{\partial x} \frac{\partial g}{\partial p}-\frac{\partial f}{\partial p} \frac{\partial g}{\partial x}
$$

and it is such that

$$
\frac{d f}{d t}-\frac{\partial f}{\partial t}=\{f, \mathcal{H}\}
$$

holds. Let us compute the expectation of the left hand side,

$$
\left\langle\frac{d f}{d t}\right\rangle-\left\langle\frac{\partial f}{\partial t}\right\rangle=\left\langle\frac{\partial f}{\partial x_{k}} \dot{x}_{k}+\frac{\partial f}{\partial p_{k}} \dot{p}_{k}\right\rangle,
$$

which using Eq. 14 with $\omega=\partial f / \partial p_{k}$ can be written as

$$
\left\langle\frac{d f}{d t}\right\rangle-\left\langle\frac{\partial f}{\partial t}\right\rangle=\left\langle\frac{\partial f}{\partial x_{k}} \dot{x}_{k}-\frac{\partial}{\partial x_{k}}\left(\frac{\partial f}{\partial p_{k}}\right)-\frac{\partial f}{\partial p_{k}} \Phi^{\prime}\left(x_{k}\right)\right\rangle
$$

Now using our classical Hamiltonian (Eq. 20) we recognize its derivatives

$$
\begin{aligned}
\dot{x}_{k} & =\frac{\partial \mathcal{H}}{\partial p_{k}} \\
\Phi^{\prime}\left(x_{k}\right) & =\frac{\partial \mathcal{H}}{\partial x_{k}}
\end{aligned}
$$

and, upon replacing, we have

$$
\left\langle\frac{d f}{d t}\right\rangle-\left\langle\frac{\partial f}{\partial t}\right\rangle=\left\langle\frac{\partial f}{\partial x_{k}} \frac{\partial \mathcal{H}}{\partial p_{k}}-\frac{\partial}{\partial x_{k}}\left(\frac{\partial f}{\partial p_{k}}\right)-\frac{\partial f}{\partial p_{k}} \frac{\partial \mathcal{H}}{\partial x_{k}}\right\rangle
$$

leading finally to

$$
\left\langle\frac{d f}{d t}\right\rangle-\left\langle\frac{\partial f}{\partial t}\right\rangle=\langle\{f, \mathcal{H}\}\rangle-\left\langle\frac{\partial}{\partial x_{k}}\left(\frac{\partial f}{\partial p_{k}}\right)\right\rangle .
$$

So, in expectation we find a Poisson bracket analog with an additional term. For the particular case $f=\mathcal{H}$, we obtain

$$
\left\langle\frac{d \mathcal{H}}{d t}\right\rangle=-\left\langle\frac{\partial}{\partial x_{k}}\left(\frac{\partial \mathcal{H}}{\partial p_{k}}\right)\right\rangle
$$

which reduces to

$$
\left\langle\frac{d \mathcal{H}}{d t}\right\rangle=-\left\langle\frac{\partial \dot{x}_{k}}{\partial x_{k}}\right\rangle=0,
$$

using the centered difference [13], 


$$
\dot{a}_{i} \approx \frac{a_{i+1}-a_{i-1}}{2 \Delta t} .
$$

Therefore we have shown that, for a Hamiltonian with the form given in Eq. [20, the energy is conserved in expectation.

[1] E. T. Jaynes, Physical Review 106, 620 (1957).

[2] J. E. Shore and R. W. Johnson, IEEE Trans. Info. Theory IT-26, 26 (1980).

[3] J. Skilling, in Maximum Entropy and Bayesian methods in Science and Engineering, edited by G. J. Erickson and C. R. Smith (Kluwer Academic Publishers, 1988), pp. 173-187.

[4] E. T. Jaynes, Ann. Rev. Phys. Chem. 31, 579 (1980).

[5] G. Stock, K. Ghosh, and K. A. Dill, J. Chem. Phys. 128, 194102 (2008).

[6] H. Haken, Z. Phys. B. Cond. Matt. 63, 505 (1986).

[7] H. Ge, S. Presse, K. Ghosh, and K. Dill (2011), arXiv:cond-mat.stat-mech/1106.4212v2.

[8] A. Caticha and C. Cafaro (2007), arXiv:physics.class-ph/0710.1071v1.

[9] S. Davis and G. Gutiérrez, Phys. Rev. E 86, 051136 (2012).

[10] E. T. Jaynes, Proceedings of the IEEE 10, 939 (1982).

[11] L. Smolin, Physics Letters 113A, 408 (1986).

[12] E. Nelson, Phys. Rev. 150, 1079 (1966).

[13] Note that using one-sided differences is not appropriate here, as one obtains $\pm 1 / \Delta t$ depending on forward or backward. 